\title{
Stratospheric Winds and Rain Effect on HAPS Backhaul Link Performance
}

\author{
Lway Faisal Abdulrazak \\ Computer Science Department, \\ Cihan University Sulaimaniya Campus, \\ Sulaimani, Iraq \\ lwayfaisal_abdulrazak@yahoo.com
}

\begin{abstract}
Abstrat: today, fourth generation of mobile communication turn out to be in active mode for several countries and one of the most promisingtechnologies used for a backhaul service is High Altitude Platform Stations (HAPS). While HAPS still suffers from environment effects in stratospheric layer like rain and winds.In this research paper, a study of the effect of the positional instability of HAPS (aircraft category) on the link level performance of an International Mobile Telecommunications Advanced (IMT-Advanced) served by HAPS is presented. The analysis is performed in three different climatic conditions of Malaysia, which is a tropical country. Then two types of radio links have been analyzed firstly, the end user link which connects aerial station and end user and then, backhaul link that establishes communication between HAPS and Ground Transmission Gateway (GTG). A Problem of fluctuations in the signal level and degrading the performance level had been solved by analyze the behavior of winds in stratospheric layer. Finally, the effect of instability was given in received signal level variation and link availability or percentage outage of the link. Obtained results showed that the link availability of the backhaul link meets the ITU-R recommendations. Lately, it can be said that the results of the service obtained can be used to design the communication network with a higher level of user satisfaction.
\end{abstract}

Keywords: HAPS, IMT-Advanced, Broadcasting, Platform instability.

\section{INTRODUCTION}

Rapid development in Wireless Communication services lead to boost up the development terrestrial and satellite Communication. High bandwidth, high signal to interference level, flexibility, small loss due to free space, wide coverage, broadband capability, and ability to relocation in emergency situations, low maintenance cost are the most important factors for excellent link establishment [1]. Therefore we can say that serial stratospheric HAPS could resolve all the requirements of an excellent link establishment for fourth generation network. This type of HAPS placed in 17 to $22 \mathrm{Km}$ height in the air [2].

Some problems may appear during HAPS deployments like fixing antennas, leaking of airship technology and HAPS remote control. It is important to mention that
HAPS hasan advantage of good handover and nice cell size which are advantages over Low earth orbit and Geostationary Satellite orbit [3]. The infrastructure of HAPS will be examined with respect to the terrestrial wireless access and integrated satellite system to create a powerful system relay on advantages of both systems [4]. Integrated HAPS shows a very good coverage with a satisfying bandwidth comparing to fiber networks, even though using HAPS has facilitate many rural and suburban areas with an excellent wireless services using antenna shift phase array. A case study has been carried on HAPS with Cellular network was carried on [5].

Stratospheric winds are still mystery for researchers to emphasize on whether HAPS can provide wireless service reliably without short term interference or not during the problem of instability. Accordingly a good research material has been done to evaluate the effect of instability on wireless link in [6].

The main problem of this research, which due to rotation and rapid burst of stratospheric wind the platform might not provide acceptable or stable services to the system. This could cause platform positional instability which hampers system level performance. This instability also made the job of designing antenna for HAPS much more complicated. The flat of perpendicular movement of HAPS could be analyzed and covered in term of carrier to interference level as in [7].

Productivity of HAPS can be determined according to the directivity of the antenna array, However, the antennas should be directly connected to the earth station with a minimum frozenly zoon [8].

So, rotation and horizontal and vertical displacements are the main area of concern.As in the stratospheric region wind is a strong reason for deviation of the aerial platform, this was taken into account, along with rotational movement, in the study to find out the issues on whether the platform can provide reliable services without temporal outages. Then, the impacts of platform positional instability of HAPS on IMT-Advanced terminal and on backhaul link at $28 / 31 \mathrm{GHz}$ band were analyzed for different scenarios, different geographical locations and also for different positions of both aerial station and ground receivers [9].

This paper organized starting with introduction then, overview operations, functionality were presented in the second section. Third section describes the results and findings. Then, conclusion is well handled. 


\section{PLACEMENT ISSUES AND \\ SPECTRUM ALLOCATION:}

The high altitude of platforms proposed in this study (17 22KM) has enabled a wide cell coverage. Since wind velocity is calculated by [9] it is very obvious that the altitude 17 to $22 \mathrm{~km}$ suffers from a medium wind velocity as in Figure 1. While the velocity of winds increasing with $25 \mathrm{~km}$ and above, it is clear that wind between 17 to $25 \mathrm{~km}$ is stable [10].

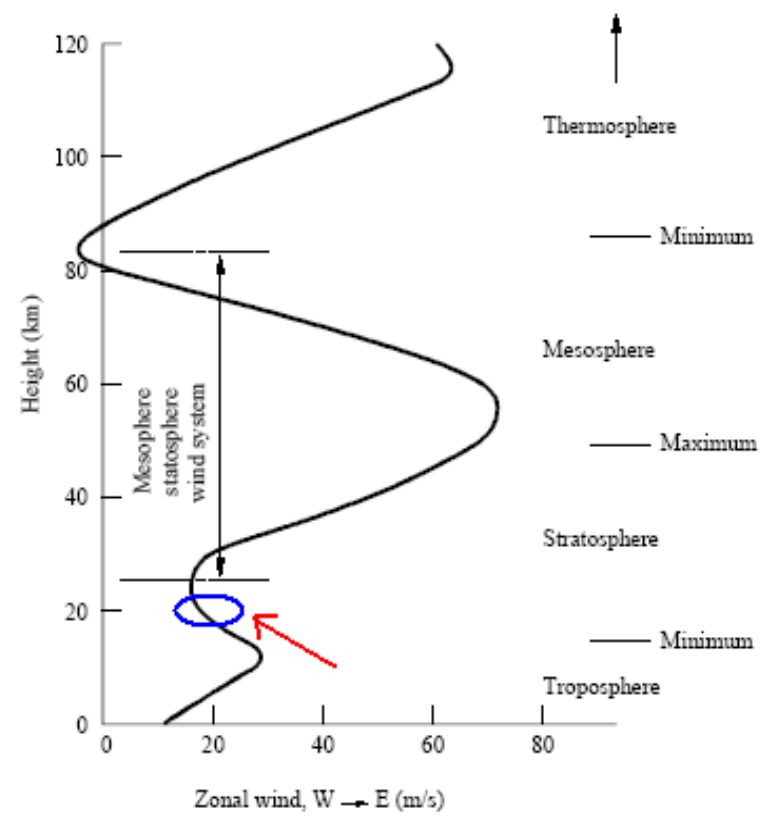

Figure 1: Wind speed correspondence to the HAPS attitude [9].

Stability in Platform is an elective matter within a range of possibilities and conditions, this paper set boundaries for a very interesting quasi-stationary link.

Designing tested for simulation scenarios has followed the ITU recommendations regarding HAPS. However, by considering a 0 access as a reference point for the aircraft over specific area, aircraft is allowed to have a smooth movement between $-200 \mathrm{~m}$ and $+200 \mathrm{~m}$ horizontally, while the height is varying between $-700 \mathrm{~m}$ and +700 m vertically [11].

It is compulsory to have a fulltime of services continuously during the simulation, to have the most accurate situations.

Regarding the matter of stabilities, navigator is playing a very important role in keeping the vertical and horizontal locations within the acceptable level vertically and horizontally. It is significant to mention that fighting altitude considered for this research paper is 20 kilometers.

Other critical considerations for this research are the aerodynamics and thermal dynamic effect on aircraft, however, air volume and temperature could reduce the link performance from space to earth and vice versa [2].
As a solution for aircraft energy, dual power provider technology was considered, mixing fuel and solar energy has eliminate the needs of carrying heavy fuel weight during the platform journey. Regarding operating frequency it was mentioned in [12] that best frequency allocation is $28 \mathrm{GHz}$ for uplink and $31 \mathrm{GHz}$ for downlink.

\section{SYSTEM PERFORMANCE}

In [4] and [13] focus was given the effect of aircraft mobility because of winds in stratospheric layer on telecommunication link degradation.

Whereas 20Mbps bandwidth has been implemented for a three cells scenarios under full mobility considerations were taken into this research by study and analysis.

Other study as in [14] covers a High Resolution Doppler Imager (HRDI) for different types of wind speed, they come up with a nice recommendations for future HAPS deployments. However, researchers in [15] mentioned that lower mesospheric temperature happened in between 10 kilometers to 115 kilometers.

Accordingly, measurements happen every 60 seconds, the standard deviation was calculated for all observed points correlated with time response within the altitude of 20 kilometers using the navigation device. Antenna specification was considered carefully to reconfigure the platform performance according to the varying position vertically and horizontally.

It was recommended by [16] that for HAPS it is better to use high frequency, in order to have free interference and wide range of frequency use, high antennas gain, reduce size and low power to decrease the overall payload. Most recommended antennas for above mentioned specifications could be Multibeam Horn Antenna (MBHA) and digital beam forming antenna (DBFA) as in table 1 [18-19].

Table 1: Main Specifications of the multibeam antenna prototypes [14].

\begin{tabular}{|l|l|l|}
\hline Parameter & MBH antenna & $\begin{array}{l}\text { Digital } \\
\text { Forming Antenna }\end{array}$ \\
\hline $\begin{array}{l}\text { Operating } \\
\text { frequency } \\
\text { range }\end{array}$ & $\begin{array}{l}\mathrm{Tx} \quad 47.2-47.5 \\
\mathrm{GHz} \quad 47.9-48.2 \\
\mathrm{Rx} \\
\mathrm{GHz}\end{array}$ & $\begin{array}{l}\mathrm{Tx} \quad 27.5-28.35 \\
\mathrm{GHz} \\
\mathrm{Rx} \quad 31.0-31.3 \\
\mathrm{GHz}\end{array}$ \\
\hline Model & $\begin{array}{l}\text { Seven corrugated } \\
\text { horns }\end{array}$ & $\begin{array}{l}16 \quad(4 \times 4) \quad \text { patch } \\
\text { array }\end{array}$ \\
\hline $\begin{array}{l}\text { Effeciency } \\
\text { Effective }\end{array}$ & $6.300 \mathrm{MHz}$ & $4 \mathrm{MHz}$ \\
$\begin{array}{l}\text { Isotropic } \\
\text { Radiation } \\
\text { Power }\end{array}$ & $11 \sim 15 \mathrm{dBW}$ \\
\hline $\begin{array}{l}\text { Gain } \\
\text { Temperature }\end{array}$ & $<-15.4 \mathrm{~dB} / \mathrm{K}$ & $-13 \sim-17 \mathrm{~dB} / \mathrm{K}$ \\
\hline bit rate & $56 \mathrm{Mb} / \mathrm{s}$ & $4 \mathrm{Mb} / \mathrm{s}$ \\
\hline $\begin{array}{l}\text { Equipment } \\
\text { Weight }\end{array}$ & $155 \mathrm{~kg}$ or less & $70 \mathrm{~kg}$ \\
\hline
\end{tabular}


As shown in Table 1; digital beam forming antenna has much wider bandwidth more stability and easier deployment than Multibeam horn. DBF antennas has meet the most suitable specifications for next generations of mobile users, however, by focusing the beam towards objectives and mulling beams in the directions of interferers

\section{END USER OR GATEWAY RECEPTION MODELING}

In case of end user, received power of user (IMTAdvanced) terminal is computed at different user locations in the ground for different aircraft positions. On the other hand, for backhaul link, received power of gateway is determined at different aircraft position and also for different polarization, namely circular, horizontal and vertical, of the transmitting HAPS antenna.

For these, the free space loss is calculated using the following formula [17],

$\mathrm{Ls}=32.45+20 \log d($ in $\mathrm{km})+20 \log f($ in $\mathrm{MHz})$

here,

Ls $=$ Free Space Loss in $\mathrm{dB}$

$d=$ Separation between receiver and transmitter in $\mathrm{km}$

$f=$ Operating frequency in $\mathrm{MHz}$.

Then, the received power at receiver is calculated by [16],

$$
\begin{aligned}
& \mathrm{Pr}=\mathrm{Pt}+\mathrm{Gt}+\mathrm{Gr}-\mathrm{Ls}-A \\
& \mathrm{Pr}=\mathrm{EIRP}+\mathrm{Gr}-\mathrm{Ls}-A
\end{aligned}
$$

here,

EIRP $=$ Effective Isotropic Radiated Power in $\mathrm{dBm}$.

$\mathrm{Pr}=$ Power at receiver in $\mathrm{dBm}$,

$\mathrm{Pt}=$ Transmitted power in $\mathrm{dBm}$,

$\mathrm{Gt}=$ Gain at tranmitter,

$\mathrm{Gr}=$ Gain at receiver $\mathrm{dB}$,

$A=$ Attenuation due to rain in $\mathrm{dB}$,

Carrier to Noise Ratio (CNR) is calculated for each received power for both end user link and backhaul link. Noise is calculated using [17]

$\mathrm{N}=\mathrm{k} * \mathrm{~T} * \mathrm{~B} * \mathrm{~F}$

here,

$\mathrm{N}=$ Noise

$\mathrm{k}=$ Boltzmann's Constant

$\mathrm{T}=$ Temperature in Kelvin

$\mathrm{B}=$ Channel Bandwidth

$\mathrm{F}=$ Receiver noise figure.

Availability of the link in computed in percentage of time based on the CNR for backhaul link. It shows how much time the link will be unavailable in a total calendar year.

\section{END-USER LINK}

The HAPS network architecture is described using Figure 2 and Figure 3. In Figure 2, four positions of the HAPS circular corridor have been highlighted - A, B, C, and $\mathrm{D}$. The black numbers indicate the ground distances from center of the HAPS rotation circle in ground to a particular position where the user might be situated. The blue numbers refer to the aerial distance between the haps and the particular user location. Figure 3 delineates the situation of user receiving different signal strength at different location of HAPS.

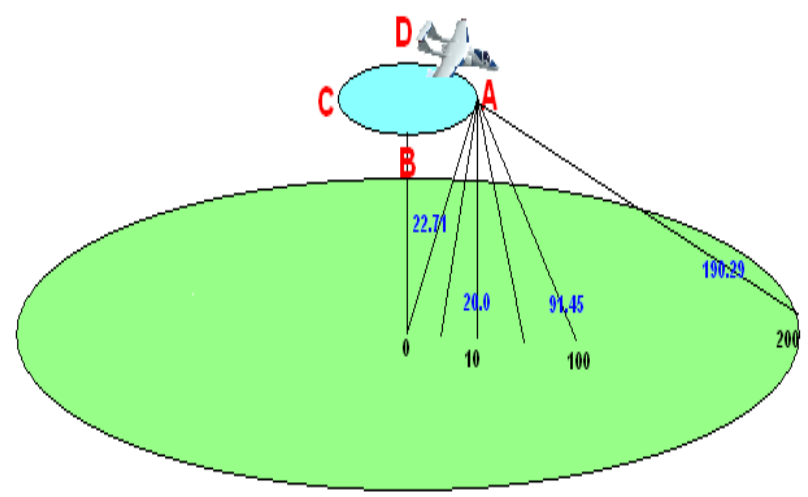

Figure 2: HAPS movement for user at different locations.

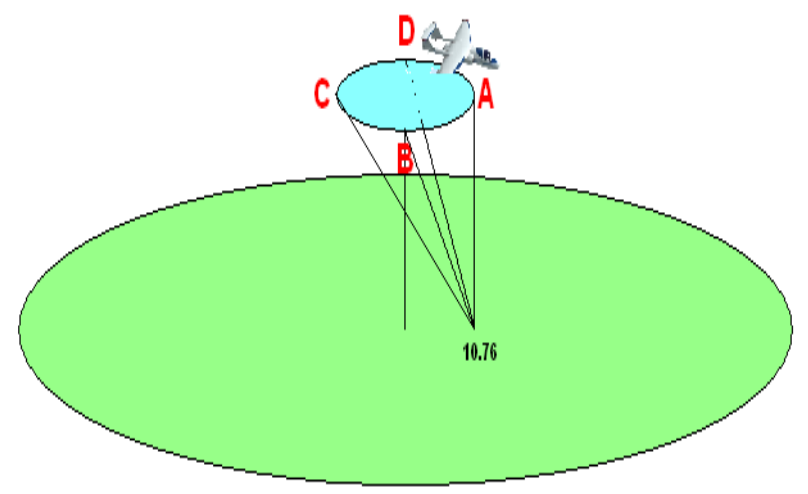

Figure 3: HAPS movement for user at specific location

At first the different values of CNR, that the receiver will get, at different positions has been determined for a single instance of HAPS position. The summarized result is shown in Figure 4. 


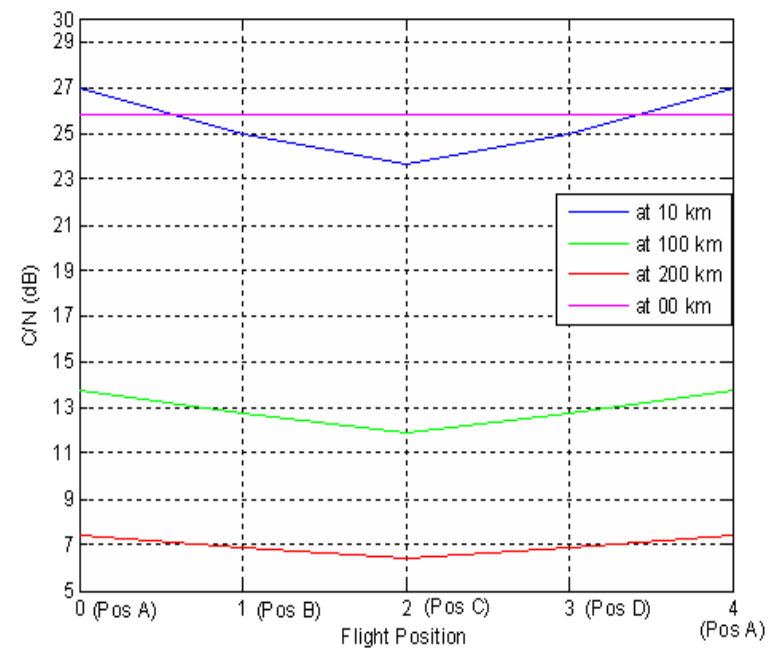

Figure 4: Comparison of CNR when receiver at different ground positions.

Here, it can be noted that when the receiver is at $00 \mathrm{~km}$, which means at center of the HAPS circle in ground, there is no fluctuation in the received signal for all the flight positions from $A$ to $D$. It has a constant value of around $26 \mathrm{~dB}$. Then, when receiver is at $10 \mathrm{~km}$ from center, it experiences the most fluctuations of about $3 \mathrm{~dB}$ due to circular rotation of HAPS. At flight position A, it has best value of $27 \mathrm{~dB}$ and at flight position $\mathrm{C}$ it faces lowest CNR o $24 \mathrm{~dB}$.

After that, in case of receiver at $100 \mathrm{~km}$, the total fluctuation of CNR among different flight positions decreases to $2 \mathrm{~dB}$. But there is a significant decrease of the total signal strength, which is now about $13 \mathrm{~dB}$. Then, for $200 \mathrm{~km}$, the total fluctuation of CNR among different flight positions is now less than $1 \mathrm{~dB}$. Subsequently, the total signal strength went down to around $7 \mathrm{~dB}$. Lastly, it should be noted that, the difference in CNR between the center of the circle and edge of the circle (at $200 \mathrm{~km}$ from center) in all flight positions is approximately $20 \mathrm{~dB}$.

The effect of horizontal and vertical fluctuation of HAPS is summarized in Figure 5, Figure 6, Figure 7 and Figure 8. To describe the phenomena properly 4 ground distances have been chosen, namely $00 \mathrm{~km}, 10 \mathrm{~km}, 100$ $\mathrm{km}$ and $200 \mathrm{~km}$ respectively.

In Figure 5, the effect is analyzed when the receiver is at $00 \mathrm{~km}$ ground distance, which means at the center of the HAPS rotational circle in ground. As the receiver is at center, there is no fluctuation in received signal for change in altitude or radius. Here, fluctuation in altitude generates more CNR variation then variation in flight path radius. But, both of these values are well below 1 $\mathrm{dB}$.

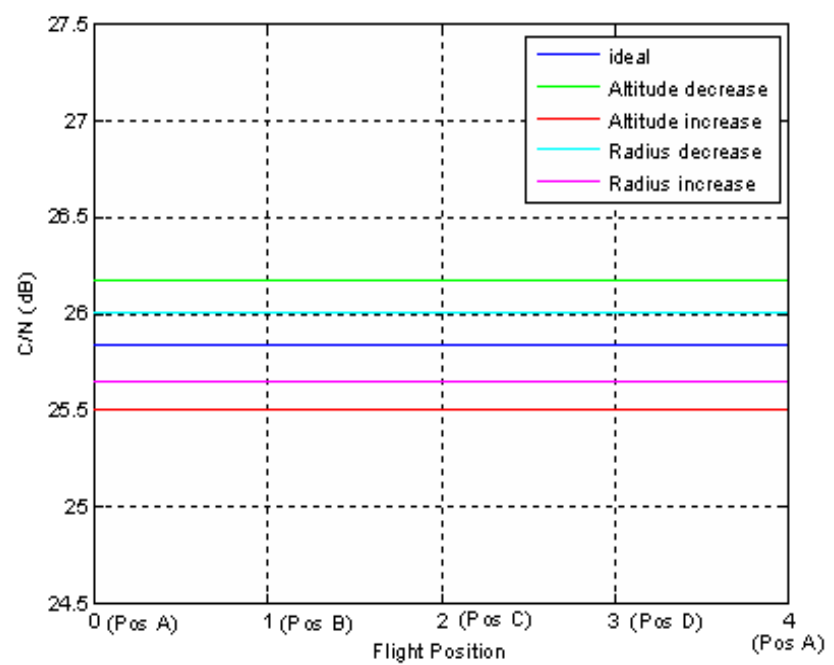

Figure 5: Altitude and radius fluctuation when receiver at $00 \mathrm{~km}$

In case of Figure 6 the receiver is at $10 \mathrm{~km}$ ground distance that means $10 \mathrm{~km}$ from center of the circle at ground. Throughout the circulation, altitude variation produce CNR difference of around $3.5 \mathrm{~dB}$ - lower value in position $\mathrm{C}$ and higher value in position $\mathrm{A}$. Radius fluctuation is not significant in position $\mathrm{A}$, but reaches the same value of $3.5 \mathrm{~dB}$ in position C. So, at $10 \mathrm{~km}$ ground distance, the receiver will face significant CNR variation in received signal.

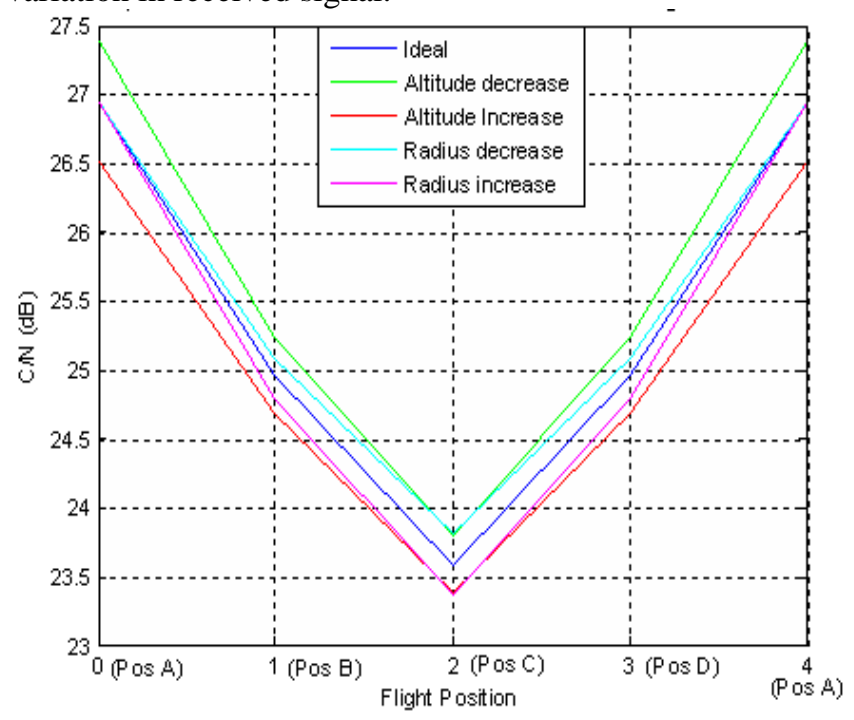

Figure 6: Altitude and radius fluctuation when receiver at $10 \mathrm{~km}$.

Figure 7 describes the situation when receiver is at 100 $\mathrm{km}$ ground distance, which means $100 \mathrm{~km}$ from the center of the circular corridor at ground. It is clear that, at this distance the altitude and radius fluctuation don't have significant impact on CNR. The highest variation in CNR is approximately $1.5 \mathrm{~dB}$ from position $\mathrm{A}$ to $\mathrm{C}$. 


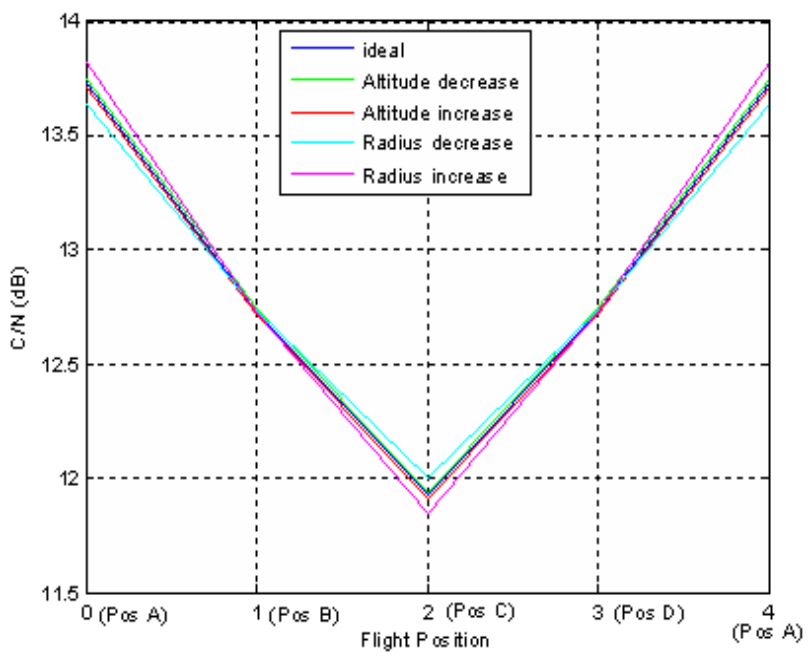

Figure 7: Altitude and radius fluctuation when receiver at $100 \mathrm{~km}$.

Then, Figure 8 demonstrates the effects when receiver is at $200 \mathrm{~km}$ ground distance, which means at the edge of coverage area. Here, the effect is almost similar as for $100 \mathrm{~km}$. The only difference is that the overall CNR fluctuation is less than $1 \mathrm{~dB}$.

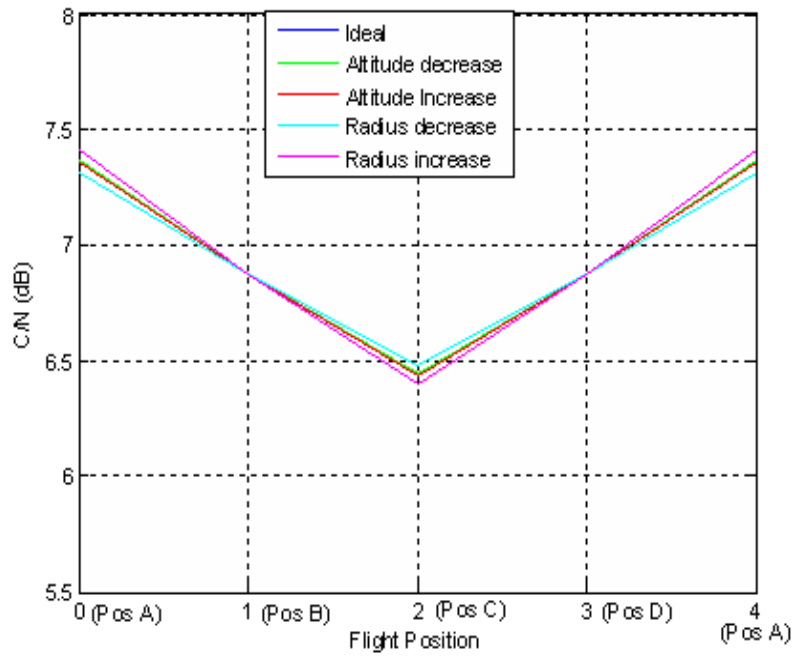

Figure 5.7: Altitude and radius fluctuation when receiver at $200 \mathrm{~km}$.

From the above results, a number of points can be extracted. Firstly, the radius and altitude fluctuation creates significant CNR variation if the receiver is closer to the center of HAPS circular corridor in ground, except the center itself. Then, when the receiver is located at long distances from center the effect of fluctuation don't have significant impact on the received signal, only the rotational effect remains. Lastly, when the receiver is close to the center, altitude fluctuation has higher impact than radius fluctuation in variation of CNR.

\section{BACKHAUL LINK}

The link availability of backhaul link is determined according to the parameters of 3 different places. The calculated network structure for backhaul link is shown in Figure 9. Here, the GTG is located at $35.35 \mathrm{~km}$ ground distance from center.

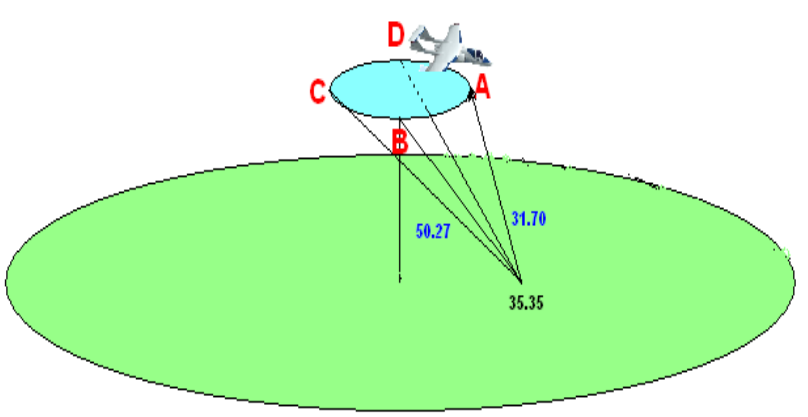

Figure 9: HAPS movement for backhaul link.

Figure 10 shows the link availability of first site for 3 different flight positions - A, B and $\mathrm{C}$ using circular polarized antenna. It is evident that, here the link can provide $99.9 \%$ of availability as it is well over the $0 \mathrm{~dB}$ CNR value. So, the percentage of outage here is $0.1 \%$, which means the link will be unavailable for 525.6 minute in a total calendar year. Now, the highest CNR variation is $8 \mathrm{~dB}$ which occurs between position $\mathrm{A}$ and C. Position $B$ gives a value in between these two.

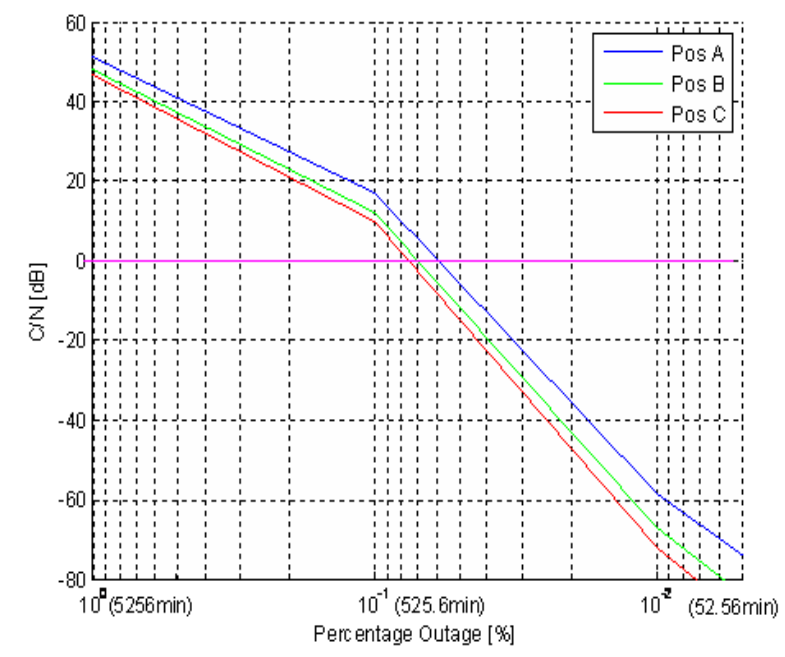

Figure 10: site 1 backhaul result for different flight position (Circular Pol.)

Then, Figure 11 shows backhaul link availability for 3 different polarization computed in flight position A. Here also, all the polarization can provide minimum availability of $99.9 \%$. Vertical polarization gives the best CNR, Horizontal polarization gives the lowest CNR and circular polarization gives a value between these two. There is a difference of $10 \mathrm{~dB}$ between received signal of horizontal and vertical polarization at $99.9 \%$ availability. 


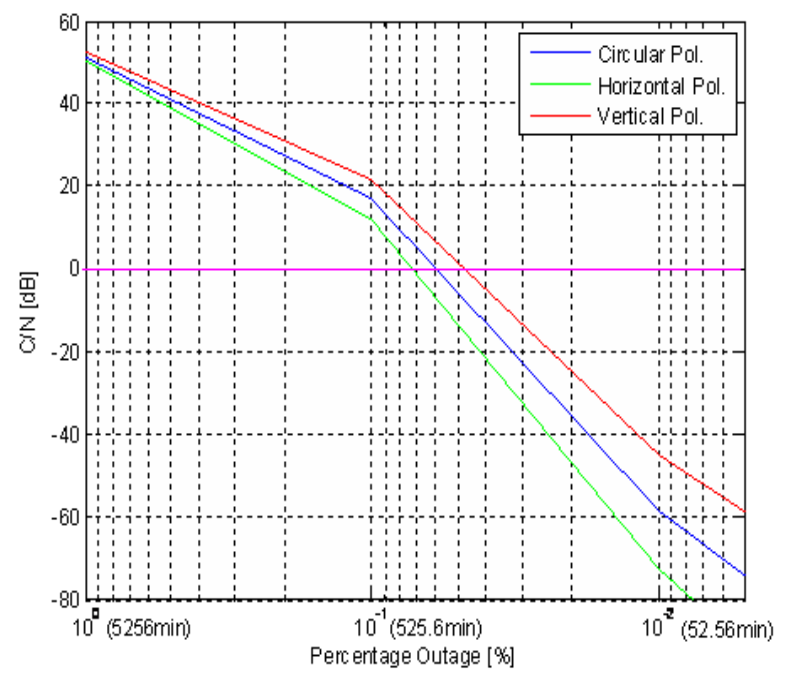

Figure 11: site1backhaul result for different Polarization (Pos. A)

Site 2 will give a measure of best possible performance of the link. In, Figure 12, the link availability is shown for 3 different flight positions - A, B and C using circular polarized antenna. It is clear that here the link can provide $99.91 \%$ of availability as it is well over the threshold CNR value. So, the percentage of outage here is $0.09 \%$, which means the link will be unavailable for 473.04 minute in a total calendar year. Now, the highest CNR variation is $7 \mathrm{~dB}$ which occurs between position A and $\mathrm{C}$.

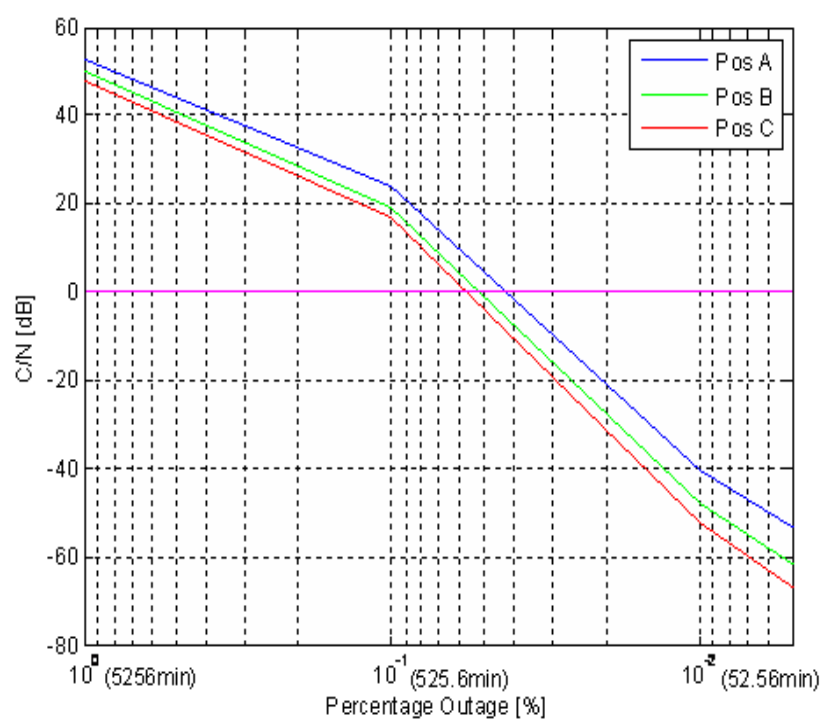

Figure 12: Site 2 backhaul result for different flight position (Circular Pol.)

Now, Figure 13 shows backhaul link availability for 3 different polarization computed in flight position A. Here, all the polarization can provide minimum availability of $99.92 \%$. So, the percentage outage in this case is $0.08 \%$, where thee link will be unavailable 420.48 minute in a year. In these case also, vertical polarization gives the best CNR, Horizontal polarization gives the lowest $\mathrm{CNR}$ and circular polarization gives a value between these two. There is a difference of $9 \mathrm{~dB}$ between received signal of horizontal and vertical polarization at $99.92 \%$ availability.

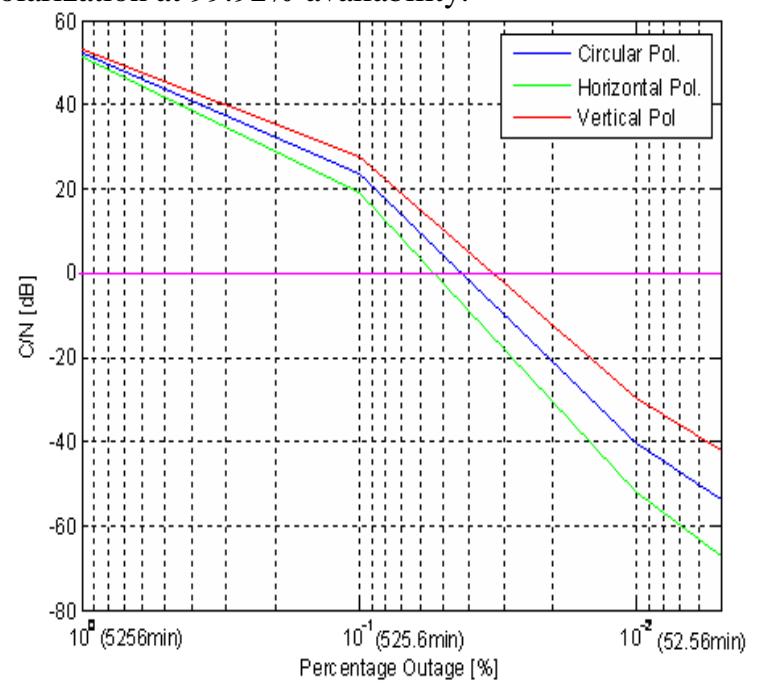

Figure 13: site 2 backhaul result for different

Polarization (Pos. A)

Site 3 is the place which experiences most rainfall. It will give a measure of worst possible performance of the link. In, Figure 14, the link availability of site 3 is shown for 3 different flight positions - A, B and C using circular polarized antenna. It is shown that here the link can provide $99.8 \%$ of availability as it is well over the 0 $\mathrm{dB}$ CNR value. So, the percentage of outage here is $0.2 \%$, which means the link will be unavailable for 1051.2 minute in a total calendar year. Here, the highest CNR variation is $7 \mathrm{~dB}$ which occurs between position $\mathrm{A}$ and $\mathrm{C}$.

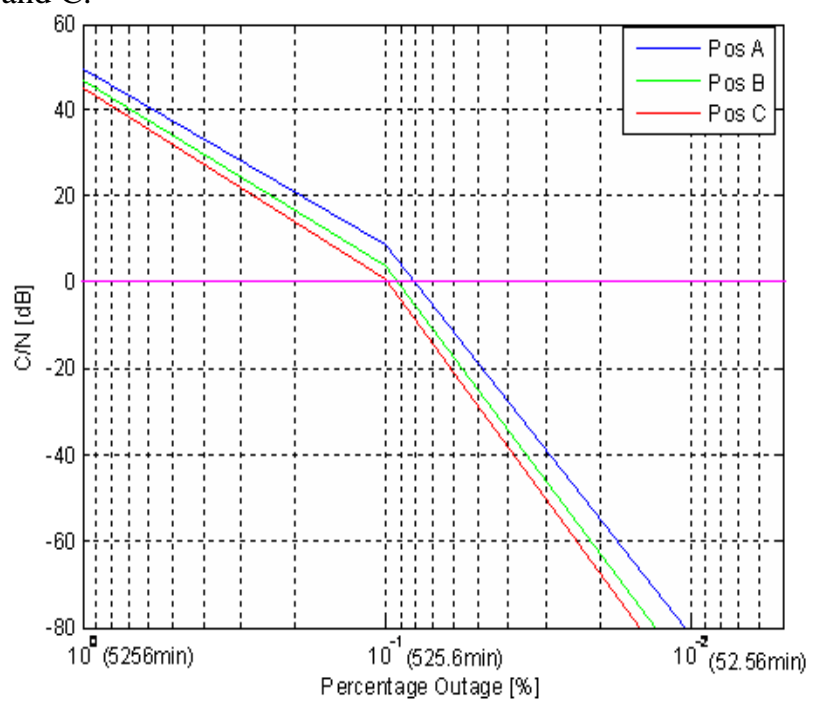

Figure 14: Site 3 backhaul result for different flight position (Circular Pol.) 
Now, Figure 15 shows backhaul link availability for 3 different polarization computed in flight position $\mathrm{A}$. Here, all the polarization can provide minimum availability of $99.8 \%$. So, the percentage outage in this case is $0.2 \%$, where thee link will be unavailable 1051.2 minute in a year. In these case also, vertical polarization gives the best CNR, Horizontal polarization gives the lowest CNR and circular polarization gives a value between these two. There is a difference of $9 \mathrm{~dB}$ between received signal of horizontal and vertical polarization at $99.8 \%$ availability.

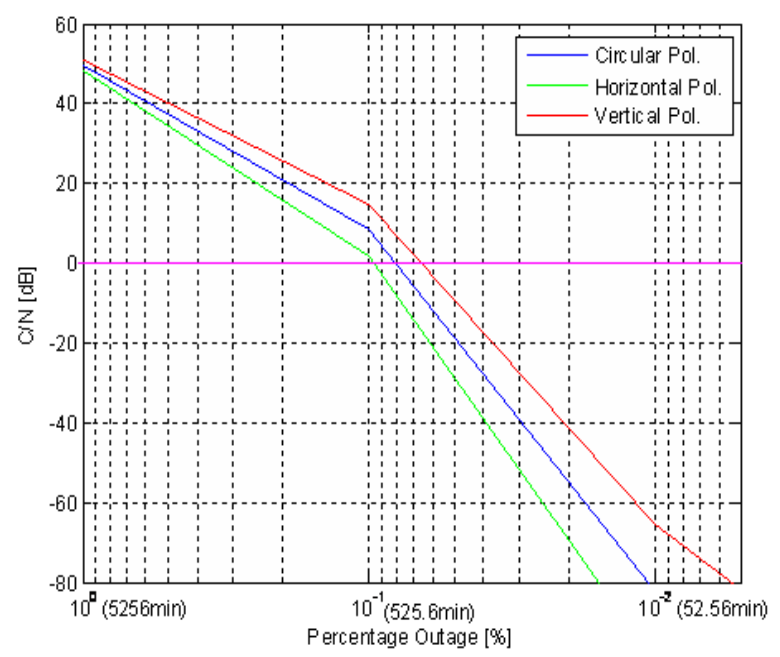

Figure 15: Site 3 backhaul result for different

Polarization (Pos. A)

As Site 2 and 3 are the places where two most diverse rainfalls are experienced, so it can be said that, in this scenario link availability between $99.8 \%$ and $99.92 \%$ can be provided. This also indicates that, the duration of backhaul link unavailability will be between 473.04 minute and 1051.2 minute. This clearly satisfies the ITU-R recommendation of minimum $99.4 \%$ [3].

In case of antenna polarization, vertical polarization always gave better result than other two. So, polarization diversity can be used for better performance. If the atmosphere normal, then either horizontal or circular polarization can be used. Then in case of heavy rain or other natural disturbances, it can be switched to vertical polarization.

\section{Conclusion}

The CNR variation from center to the edge of servicing area is around $20 \mathrm{~dB}$ for IMT-Advanced end user. Moreover, if a user is located at a fixed position it can suffer from maximum $3 \mathrm{~dB}$ variation in the received signal due to the rotation and fluctuation. Furthermore, users close to the center suffer more variation in signal than users located at long distances and altitude fluctuation degrades the signal more than radius fluctuation. The backuhaul link also suffers from a number other effects besides the above stated ones, such as rain attenuation. In this case, signal levels were determined in three different places with different rainfall statistics - Site 1, 2 and 3. It is observed that even in the worst case condition (in Site 3) the link can provide $99.8 \%$ link availability, which means the link may be down for around 1051 minute in a total year. This statistics is higher than that of $99.4 \%$, which is proposed by ITU-R. On the other hand, in best case condition, (in Site 1) calculation showed that, even $99.91 \%$ of link availability can be achieved. Last but no least, research on different antenna polarization reveled that, vertical polarization, which gave the best performance, can be used with either circular or horizontal polarization in polarization diversity to obtain optimum system performance. Lastly, it can be said that, the result of the service obtained can be used to design the communication network with higher level of user satisfaction. These findings might also be useful for the design team of HAPS to come up with more robust aerial stations and better ways of stabilizing mechanism.

\section{REFERENCE}

[1] L.F. Abdulrazak, T.A.Rahman, S.K. Abdul Rahim and M. Yousif, " Study HAPS Interference Power to Noise Level Ratio of Fixed Services and Related Separation Distance, " Information Sciences Signal Processing and their Applications (IS SPA), 2010, pp. 690-693.

[2] S. Karapantazis, N. Pavlidou, "Broadband communications via high-altitude platforms: a survey", IEEE Commun. Surveys and Tutorials, vol. 7, no. 1, pp. 2-31, 2005.

[3] D. I. Axiotis, M. E. Theologou, E. D. Sykas, "The effect of platform instability on the system level performance of HAPS UMTS", IEEE Commun. Lett., vol. 8, no. 2, pp. 111-113, 2004.

[4] C. Feng, R. Wang, Z. Bian, T. Doiron, J. Hu, "Memory dynamics and transmission performance of bundle protocol (BP) in deep-space communications", IEEE Trans. Wireless Commun., vol. 14, no. 5, pp. 2802-2813, May 2015.

[5] N. Baccour, A. Koubâa, H. Youssef, and M. Alves, "Reliable Link Quality Estimation in Low-Power Wireless Networks and its Impact on TreeRouting," Ad Hoc Networks, vol. 27, no. C, pp. 125,2015 .

[6] A. Mohammed, A. Mehmood, F.-N. Pavlidou, M. Mohorcic, "The role of High-Altitude Platform Station (HAPS) in the global wireless connectivity", Proceedings of the IEEE, vol. 99, no. 11, pp. 1939-1953, 2011.

[7] Recommendation ITU-R M.1456, Minimum Performance Characteristics and Operational Conditions for High Altitude Platform Stations providing IMT-2000 in the Bands $1885-1980 \mathrm{MHz}$, $2010-2025 \mathrm{MHz}$ and $2110-2170 \mathrm{MHz}$ in the Regions I and 3 and 1885-1980 MHz and 2110$2160 \mathrm{MHz}$ in Region 2, International Telecommunications Union, 2000.

[8] T.C. Tozer, D. Grace, "High-Altitude Platforms for Wireless Communications", vol. 13, no. 3, June 2001. 
[9] ITU-R Study Groups. Specific attenuation model for rain for use in prediction methods. Rec. ITU-R P.838-3. 2005.

[10] Colozza, A. and Dolce, J. L.High-altitude, LongEndurance airships for coastal surveillance. NASA/TM-2005-213427, National Aeronautics, and Space Administration. February 2005.

[11] ITU-R Study Groups. Technical and operational characteristics for the fixed service using high altitude platform stations in the bands 27.5-28.35 GHz and 31-31.3 GHz. Rec. ITU-R F.1569, 2002.

[12] ITU-R Study Groups. Propagation data and prediction methods required for the design of terrestrial line-of-sight systems. Rec. ITU-R P.53011. 2005.

[13] B. Ku, D. Ahn, D. Baek, K. Park, S. Lee, " Degradation Analysis of User Terminal EIRPand G/T due to Station-Keeping Variation ofStratospheric Platform", ETRI Journal. March 2000. 22(1): 12-19.

[14] V. Sgardoni, A. R. Nix, "Raptor Code-aware Link Adaptation for Spectrally Efficient Unicast Video Streaming over Mobile Broadband Networks", IEEE Transactions on Mobile Computing, Vol: 14, N(2), pp: 401-415, Feb. 2015.

[15] ITU-R Study Groups. Preferred characteristics of systems in the fixed service using High Altitude Platforms operating in the bands $47.2-47.5 \mathrm{GHz}$ and 47.9-48.2 GHz. Rec. ITU-R F.1500, 2000.

[16] R. Miura, M.Suzuki, "Preliminary Flight Test Program onTelecom and Broadcasting Using High Altitude Platform Stations", Wireless Pers. Communication, An Int'l. J. Kluwer Academic Publishers. Vol:24, N (2): PP: 341-361, Jan 2003.

[17] B. El-Jabu, R. Steele, "Cellular Communications Using Aerial Platforms", IEEE Transaction on Vehicular Technology, vol. 50, no. 3, pp. 686-700, May 2001.

[18] M. Oodo, R. Miura, Y. Hase, T. Inaba, T.

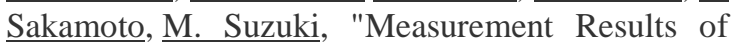
digital beamforming array antenna on-board stratospheric platform in the band $31 / 28 \mathrm{GHz}$ ", The 5 th international symposium on wireless personal multimedia communications. 2002. vol. 2, pp. 435439.

[19] Rappaport, T.S. Wireless Communications: Principles and Practice. 2nd Edition. PEARSON Education. 2002.

\section{Biography}

Lway F. Abdulrazakwas born in Iraq in 1982. He received the B.Sc in Electrical and Telecommunication Eng. From Omer AlmokhtarUniversity, Libya in 2005 with 2nd honor degree; and he received his $\mathrm{MSc}$ and $\mathrm{PhD}$ degree in Electrical Electronics and Telecommunication Engineering from Universiti Teknologi Malaysia, Malaysia, in 2007 and 20011, respectively. Currently he is a lecturer at Cihan University - Sulaimanyia Campus, Iraq. His research interests include IMT-Advanced, Digital signal processing, Radio Propagation and Wireless interference analysis. 